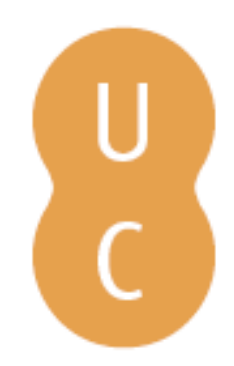

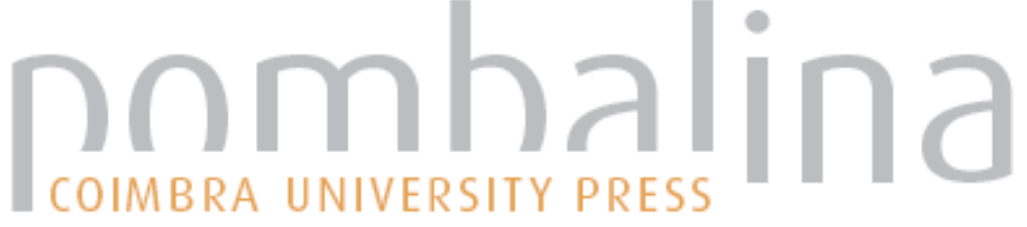

\section{Uso de dados VANT na vetorização de rodovias}

Autor(es): $\quad$ Silva, Claudionor; Gonçalves, José Alberto; Bastos, Luísa

Publicado por: Imprensa da Universidade de Coimbra

URL

persistente:

URI:http://hdl.handle.net/10316.2/37098

DOI:

DOI:http://dx.doi.org/10.14195/978-989-26-0983-6_45

Accessed : $\quad$ 26-Apr-2023 12:24:38

A navegação consulta e descarregamento dos títulos inseridos nas Bibliotecas Digitais UC Digitalis, UC Pombalina e UC Impactum, pressupõem a aceitação plena e sem reservas dos Termos e Condições de Uso destas Bibliotecas Digitais, disponíveis em https://digitalis.uc.pt/pt-pt/termos.

Conforme exposto nos referidos Termos e Condições de Uso, o descarregamento de títulos de acesso restrito requer uma licença válida de autorização devendo o utilizador aceder ao(s) documento(s) a partir de um endereço de IP da instituição detentora da supramencionada licença.

Ao utilizador é apenas permitido o descarregamento para uso pessoal, pelo que o emprego do(s) título(s) descarregado(s) para outro fim, designadamente comercial, carece de autorização do respetivo autor ou editor da obra.

Na medida em que todas as obras da UC Digitalis se encontram protegidas pelo Código do Direito de Autor e Direitos Conexos e demais legislação aplicável, toda a cópia, parcial ou total, deste documento, nos casos em que é legalmente admitida, deverá conter ou fazer-se acompanhar por este aviso. 


\section{$\forall$ \\ TAS DAS I JORNADAS LUSÓFONAS DE CIÊNCIAS E TECNOLOGIAS DE INFORMAÇÃO GEOGRÁFICA}

Editores

José Gomes dos Santos

Cidália Fonte

Rui Ferreira de Figueiredo

Alberto Cardoso

Gil Gonçalves

José Paulo Almeida

Sara Baptista 


\title{
ARTIGO 45
}

\section{USO DE DADOS VANT NA VETORIZAÇ̃̃O DE RODOVIAS}

\author{
SILVA, Claudionor ${ }^{1}$; GONÇALVES, José Alberto ${ }^{2}$ E BASTOS, Luísa ${ }^{2}$
}

\begin{abstract}
${ }^{1}$ Universidade Federal de Uberlândia, Instituto de Geografia, Campus Monte Carmelo; Rua Goiás, 2000, 38500-000, Monte Carmelo/MG, Brasil; Tel.: +34-3842-8751; Fax: +34-3842-8751; email: crs@ig.ufu.br 2 Faculdade de Ciências da Universidade do Porto; Departamento de Geociências, Ambiente e Ordenamento do Território; Rua do Campo Alegre, 687, 4169-007 Porto, Portugal; Tel.: +351-22-0402000; Fax: +351-22-0402009; email: jgoncal@fc.up.pt
\end{abstract}

\section{RESUMO}

A extração manual de rodovias é um processo exaustivo e oneroso. Várias abordagens têm sido propostas no intuito de automatizar ou semi-automatizar essa tarefa. Nesse trabalho, é proposto um método automático de extração de rodovia em dados (imagem e altimetria) obtidos com câmeras de pequeno formato em VANT e em aeronave convencional. Tanto a automatização da tarefa quanto o conceito dos dados VANT é de redução de trabalho, custo e tempo. O fundamento do método é a deteção de segmentos de reta pertencentes a uma rodovia (semente) e uma posterior busca sequencial, a partir da semente, de feições lineares que representam o eixo de rodovias. A deteção de sementes tem por base o cluster k-média e a regressão linear enquanto a busca sequencial faz uso de uma função de aptidão composta por quatro parâmetros. A validação dos resultados foi realizada com os índices: completeza, correção e RMS, medidos em uma imagem de referência, vetorizada manualmente. A proposta é promissora tendo em vista a qualidade dos resultados obtidos e a economia proporcionada em todo o processo.

\section{PALAVRAS-CHAVE}

VANT, Extração de rodovias, MDE, K-média e Regressão linear. 
Atas das I Jornadas Lusófonas de Ciências e Tecnologias de Informação Geográfica, Sessão 10, Artigo 45

Uso de dados VANT na vetorizacão de rodovias

Claudionor Silva, José Gonçalves \& Luísa Bastos

USE OF UAV DATA IN THE VECTORIZATION OF ROADS

\begin{abstract}
Manual digitising of roads is an expensive and time consuming task. Several approaches have been proposed in order to automate this task. In this work we propose an automated method of extracting roads from data (images and elevation data) obtained with small format cameras on board of a UAV or a small manned plane. Both the UAV data collection and the processing have the goal of reducing the amount of work, time and costs. The basis of the method is the detection of line segments belonging to roads (seed) and a sequential search, from the seed, of linear features that represent the axis of the roads. The seed detection is based on k-mean cluster and linear regression, while the sequential search uses a cost function composed of 4 parameters. The validation of results was done by completeness, correction and RMS indices, measured in a reference image, manually digitised. This is a promising method, with a good quality result and economy achieved in the process.
\end{abstract}

\title{
KEYWORDS
}

UAV, Road extraction, MDE, K-mean e Linear regression.

\section{INTRODUÇÃO}

Os avanços tecnológicos ocorridos especialmente na indústria de eletrônicos, que permitem acesso, manipulação e armazenamento de grande volume de dados, bem como fácil acesso à internet, tem popularizado o uso de mapas cartográficos digitais. Nesta era digital, o mapeamento rodoviário tem ganhado destaque devido à sua multiplicidade de uso e aplicações. Contudo, o método convencional de vetorizar manualmente os mapas rodoviários é um processo que não atende, em sua totalidade, à demanda supracitada. Uma opção usada para reduzir tempo, dinheiro e trabalho, nesse processo, é fazendo uso de imagens digitais. São dados de fácil acesso nos dias atuais, de custo relativamente baixo, cobrem grandes áreas e podem apresentar boa qualidade geométrica, se corrigidas com o devido rigor.

As imagens digitais são comumente obtidas por sensores acoplados em plataformas aéreas e orbitais. Atualmente, a novidade, em termos de plataforma, é o VANT (Veículo Aéreo Não-Tripulado), que surge com a promessa de redução do tempo de coleta dos dados, melhoria nas resolu- 
ções temporal e espacial, e economia em todo o processo de mapeamento, da coleta à confeção do produto final (CHIANG et al., 2012; WALLACE et al., 2012; AGUASCA et al., 2013; CRAMER, 2013).

\subsection{Veículo aéreo não-tripulado}

A coleta rápida de informação espacial tornou-se uma tendência emergente no Sensoriamento Remoto, na Fotogrametria e em aplicações de mapeamento. Para facilitar tais aplicações, o desenvolvimento acelerado de sistemas de baixo custo, aliado à coleta de informação espacial em tempo real, tornou-se uma necessidade. O VANT, em sua proposta final, proporciona essas propriedades, tendo requisitos mínimos para descolagem e aterrizagem e manutenção muito simples. A geração de imagem VANT é um setor em tecnologia de Sensoriamento Remoto de mais rápido desenvolvimento. Essa tecnologia ganhou espaço no meio Civil, em aplicações diversas, especialmente na agricultura e em monitoramento do meio ambiente (CHIANG et al., 2012; LIN \& SARIPALLI, 2012; GONÇALVES \& HENRIQUES, 2013).

Atualmente, a aplicação de dados VANT no mapeamento vem sendo testada e aprimorada em vários quisitos. Por exemplo, Du e Gao (2012) testaram um INS (Inertial Navegation System), que é um sistema necessário em VANTs aplicados ao mapeamento. A proposta é um algoritmo para identificação e deteção de deslocamento de ciclos nas frequências GPS (L1 e L2), que ocorrem devido à dinâmica, obstruções de sinal e da baixa elevação dos satélites. Hruska et al. (2012) além de analisarem a precisão de um sistema inercial (INS) no processo de georreferenciamento de imagens, fizeram a calibração radiométrica de um espectrômetro imageador, ambos embarcados em um VANT.

Dada a complexidade na gestão de estradas, onde comumente um limitado número de veículos é monitorado, Skoglar et al. (2012) propuseram o uso de um VANT, com sensor infravermelho, para suprir os recursos limitados dos sensores usados atualmente em sistemas de vigilância, que geralmente não cobrem grandes áreas e são de baixa resolução. 


\subsection{Extração de rodovias}

Com o surgimento das novas tecnologias do mapeamento móvel, câmeras fotogramétricas obliquas e dos VANTs, as técnicas desenvolvidas para extração de feições em imagens digitais tem ganhado destaque nos campos da Visão Computacional, Sensoriamento Remoto e Fotogrametria; porém o uso de dados VANTs como suporte as essas técnicas é ainda discreto. Em extração de rodovias/estradas, por exemplo, são poucos os estudos e testes com esse produto (LINGUA et al., 2009).

As técnicas propostas para extração de rodovias podem ser classificadas em dois grupos: semi-automatica (com intervenção humana) e automatica (sem intervenção). O objetivo fundamental dessas técnicas é reduzir os custos e o tempo de processamento na execução dessa tarefa. A extração de rodovias em imagens digitais tem sido abordada de diferentes formas, com diferentes algoritmos e variados tipos de dados; contudo, são poucos os métodos propostos para extrair rodovias a partir de dados VANT, que ainda apresentam limitações relativas à resolução, IMU e sistemas GPS (BAUMGARTNER et al., 1999; HU and TAO, 2007; LIN \& SARIPALLI, 2012).

Dados coletados com um VANT foram utilizados por Zhang (2008) como parte de um projeto de monitoramento das condições das estradas não pavimentadas nos EUA. O sistema foi equipado com um controlador autônomo, um GPS/IMU e um sensor magnético para detetar a posição, a velocidade e atitude do helicóptero. Um computador foi usado para comunicar com o helicóptero, em tempo real, para monitorar parâmetros de voo e enviar comandos de controle. O sistema de processamento de dados proposto inclui o modelo da superfície da estrada 3D digital, geração de ortoimagem, extração automática de feições e avaliação das condições da estrada. Os resultados parciais apontaram o sistema como promissor.

Um algoritmo de limiar adaptativo, baseado em histograma de imagens VANT, é proposto por Lin e Saripalli (2012), para detetar possíveis regiões de rodovias. O método proposto para detetar segmentos de retas faz uso da Transformada Hough e segmentação. Foram usadas imagens coletadas em áreas ruais e urbanas, com estradas que apresentavam características 
bem distintas. Os resultados indicaram sucesso na deteção em 97\% das imagens testadas.

Um projeto de VANT é desenvolvido por Feng et al. (2009), apresentando um sistema de mapeamento que capta a imagem e determina a geometria da estrada e localiza veículo em tempo real. A versatilidade do VANT permitiu a realização de reparos na cobertura aérea, quando detetado algum espaço sem imageamento. Os experimentos mostraram que o sistema funcionou bem no mapeamento de veículos em estrada, em tempo real.

\subsection{Análise de Agrupamentos}

Análise de agrupamento de dados é uma técnica de agrupamento de objetos possuidores de características intrínsecas semelhantes. É descrita na literatura com várias denominações, tais como: clustering, cluster analysis, segmentation analysis, taxonomy analysis ou unsupervised classification (GAN et al., 2007). Entre os algoritmos que descrevem a técnica de agrupamento, o k-médias e o isodata são os mais utilizados.

O k-médias é um método de agrupamento particionado ou não-hierárquico, onde o número $\mathrm{k}$ de grupos é fixado, não impondo uma hierarquia à estrutura. No método, uma matriz $\mathrm{X}$, de $\mathrm{n}$ x $\mathrm{p}$ pontos/observações, é particionada em $\mathrm{k}$ grupos de elementos semelhantes entre si, minimizando, iterativamente, a soma das distâncias dos pontos/observações ao centróide de cada cluster. $\mathrm{O}$ algoritmo k-médias pode ser compreendido pelas etapas descritas no fluxograma da Figura 1.

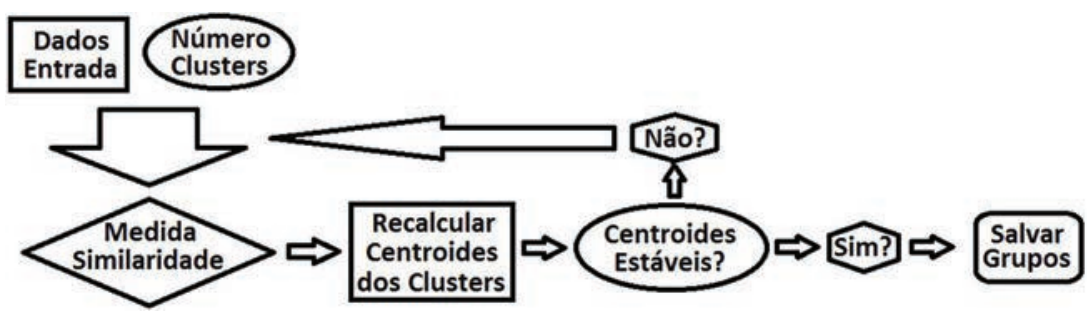

Figura 1 - Fluxograma do algoritmo k-médias

O cálculo do centroide é realizado conforme Equação 1. 


$$
\left\{\begin{array}{l}
x_{c j}=\frac{1}{n} \sum_{i=1}^{n} x_{i} \\
y_{c j}=\frac{1}{n} \sum_{i=1}^{n} y_{i}
\end{array}\right.
$$

onde $x_{i}$ e $y_{i}$ são as coordenadas da observação/ponto i; $x_{c j}$ e $y_{c j}$ são as coordenadas do centróide do cluster j; n é o total de observações/ pontos existentes no cluster $\mathfrak{j}$.

Qualquer modelo de medida de distância de similaridade pode ser usado no k-média. Devido a simplicidade de cálculo, a distância Euclidiana ao quadrado (Equação 2) é a mais comumente usada.

$$
d E^{2}=\left(x_{i}-x_{c j}\right)^{2}+\left(y_{i}-y_{c j}\right)^{2}
$$

Além dos $\mathrm{k}$ clusters, o método retorna outras medidas como o valor dos k centroides e a soma das distâncias das observações para o centroide do cluster ao qual pertencem.

\subsection{Dados Utilizados}

Os dados foram coletados com câmeras de pequeno formato acopladas em uma aeronave convencional (Cessna da Força Aérea Portuguesa) e em uma plataforma VANT.

Os itens utilizados no voo convencional foram: um sistema inercial (Litton LN200) e GPS (dupla frequência, diferencial); uma câmera de vídeo modelo AVT Pike de resolução 5 Mpixels (2452 x 2054 pixels), com sensor de $8,46 \times 7,09 \mathrm{~mm}$ e distância focal de $8,3 \mathrm{~mm}$. O voo foi planejado com os seguintes parâmetros: velocidade de $270 \mathrm{~km} / \mathrm{h}$; altura de $900 \mathrm{~m}$. Para o processamento fotogramétrico foram extraídas frames individuais do vídeo, à cadência de 1 frame por segundo, correspondendo a uma sobreposição longitudinal de $85 \%$. A triangulação aérea foi realizada/ verificada com 10 pontos de controle.

O VANT (Figura 2) utilizado nesse estudo é um pequeno avião, com bateria elétrica, peso útil inferior a $1 \mathrm{~kg}$, que pode voar em baixas altitudes, tem completa autonomia de voo e necessita de pequeno espaço para decolagem e pouso, além de fácil manuseio. A câmera fotográfica utilizada 
é modelo Canon Ixus 220 HS, resolução de 12 Mega pixels (4000 x 3000 pixels), tamanho do sensor de 6,20 x 4,65mm e distância focal de 4,3mm.

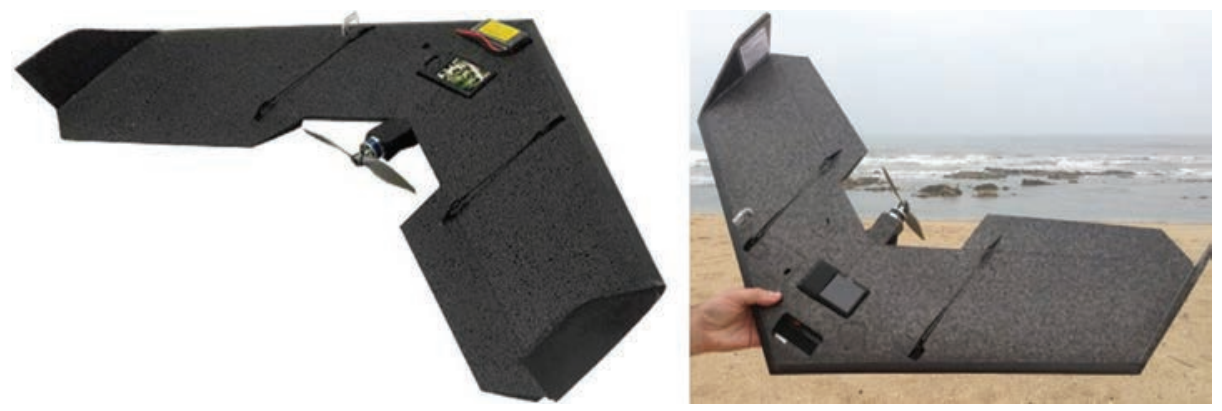

Figura 2 - Aeromodelo utilizado (Fonte: Gonçalves e Henriques, 2013.)

O voo VANT foi planejado com as seguintes características: altitude de 138 metros, velocidade de $30 \mathrm{Km} / \mathrm{h}$, obtenção de 150 fotos, resolução espacial de $4,7 \mathrm{~cm}$. Vários pontos de controle/verificação foram coletados com recetor RTK, de dupla frequência, com precisão centimétrica e observações em curto intervalo de tempo.

No processamento, muitos pontos (centenas de milhares) foram envolvidos no ajustamento dos modelos com as 150 fotos. O resíduo (RMS) medido no espaço imagem foi de 0,84 pixel. O RMS medido no georreferenciamento do MDE, utilizando os pontos de verificação e a transformação conforme 3D, foi de 0,006, 0,008 e 0,023 pixel para $\mathrm{X}, \mathrm{Y}$ e $Z$, respetivamente.

Nesse estudo foram utilizados dois recortes dos mosaicos, que apresentavam a feição de interesse (estradas/rodovias), gerados a partir dos dados VANT e da câmera de pequeno formato acoplada na aeronave convencional supracitada. A dimensão dos recortes (linhas $\mathrm{x}$ colunas) é de 563x713 e 790x816 pixels, respetivamente. As áreas testes são na região da Leiria no caso da aeronave convencional (Figura 3a) e Viana do Castelo, no caso do VANT, (Figura 3b). As resoluções das imagens são de 0,10 e $0,30 \mathrm{~m}$, respetivamente. 


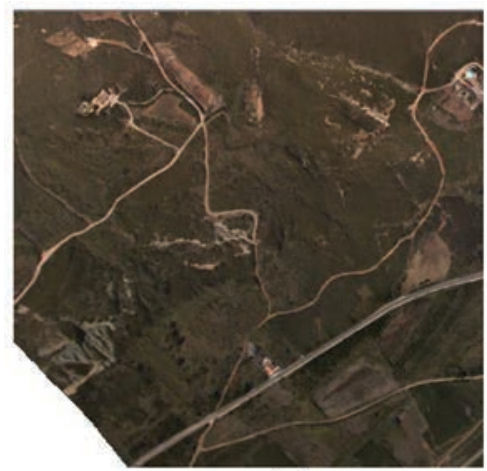

(a)

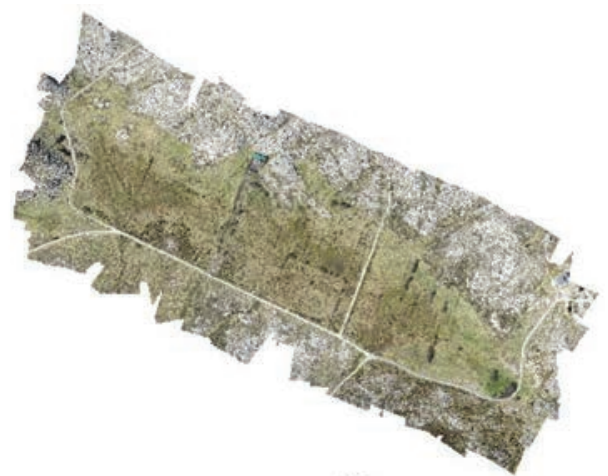

(b)

Figura 3 - Áreas Testes: a) Área 1 e b) Área 2

O processamento das fotos para geração do mosaico de ortofotos e do MDE foi realizado no software Agisoft Photoscan. Para manipular/processar os recortes das imagens foram usados os softwares MultispecW32 e ENVI 4.8. Além dos softwares comerciais, foi desenvolvido um programa para extração de rodovias, ambiente MatLab, especialmente para esse estudo.

\section{MÉTOdo}

O método proposto envolve o desenvolvimento de duas rotinas, criadas em ambiente matlab e implantadas com interface gráfica, envolvendo: a) deteção de segmentos semente, b) extração de eixos/pistas de rodovias.

\subsection{Deteção de Segmentos Semente}

O método proposto para detetar segmentos semente é baseado em regressão linear e cluster k-médias. O princípio do método é a divisão da imagem de entrada em $\mathrm{n}$ outras sub-imagens, facilitando o processamento e aumentando, nesse caso, a eficácia tanto da regressão linear quando do agrupamento k-médias. A divisão é justificada, dada que a aplicação do cluster k-médias em uma grande cena, como aquela ilustrada na Figura 3a, pode causar grande confusão espectral entre a feição rodovia (objeto de interesse) e as áreas com solo exposto, reduzindo a eficiência do método.

Primeiramente, em cada sub-imagem é aplicado o cluster k-médias, 
com um valor pré-definido, empiricamente, para k. Tendo em vista que as rodovias apresentam alto valor de reflectância (Figura 4a) e, geralmente, se destacam em relação às outras feições, em imagens de áreas rurais, o grupo/objeto correspondente ao maior centróide (Figura 4b) é então isolado (Figura 4c) para análise de pertinência ou não a uma rodovia.

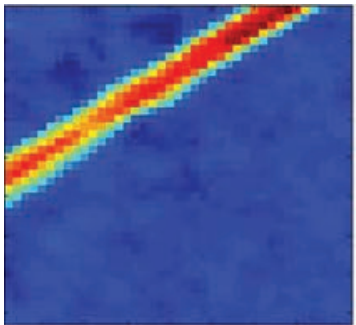

(a)

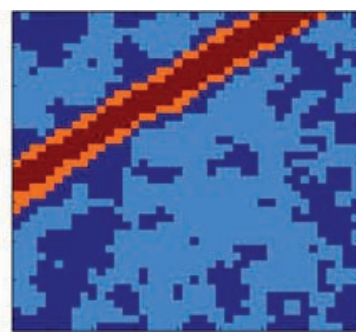

(b)

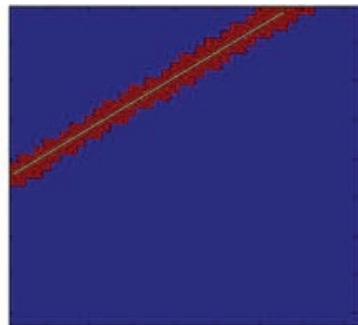

(c)

Figura 4 - Sub-imagens: a) sub-imagem original, b) k-médias da sub-imagem, c) objeto de maior centróide

Em seguida, cada segmento isolado nas sub-imagens é submetido ao processo de análise de pertinência a uma rodovia. Para realizar essa tarefa, o modelo de Regressão Linear (Equação 3) é aplicado sobre os pontos correspondentes aos objetos/segmentos isolados pelo cluster k-média.

$$
Y_{i}=\alpha+\beta \cdot X_{i}+\varepsilon_{i}
$$

onde Yi é a i-ésima variável dependente (linhas da sub-imagem); $\alpha$ é o valor de intersecção da reta de regressão com o eixo Yi; $\boldsymbol{\beta}$ é o coeficiente angular da reta de regressão; Xi é a i-ésima variável explicativa (colunas da sub-imagem) e; $\varepsilon_{i}$ é a variável dos fatores residuais e erros de medição.

A dispersão dos pontos/observações em relação à reta de regressão pode ser obtida pela medida do desvio padrão (Equação 4).

$$
\sigma=\sqrt{\frac{\sum_{i=1}^{n}\left(y_{i}-Y_{i}\right)^{2}}{n-2}}
$$

As duas intersecções da reta de regressão com a sub-imagem geram as coordenadas das extremidades dos segmentos candidatos a sementes (Figura 4c). Esse segmento é submetido ao critério de pertinência à ro- 
dovia, que é definido pelo comprimento e pela dispersão dos pontos/ observações em relação ao segmento em análise. Isto é, segmentos com tamanho maior que um limiar T e dispersão menor que um limiar T1, são classificados como sementes. O segmento ilustrado na Figura 4c é um exemplo de candidato que seria selecionado.

\subsection{Deteção de Novos Segmentos}

Com a posse de um ou mais segmentos semente, o processo de deteção e vetorização de eixos de rodovias pode ser iniciado. A deteção sequencial de segmentos, a partir de um segmento semente, tem mostrado bom desempenho (SILVA et al., 2010; SILVA \& CENTENO, 2011). Esse método toma o segmento semente por base e inicia a busca (nas duas direções, mas uma de cada vez) por outro novo segmento que pertença à mesma rodovia e que seja consecutivo e adjacente à semente.

O algoritmo trilha pelo seguinte percurso: a) partindo da semente, um segmento consecutivo, adjacente e colinear, de tamanho J (pré-definido), é projetado a vante; b) um semiarco de raio $\mathrm{J}$, correspondente a um ângulo pré-definido, é traçado tendo como bissetriz o novo segmento (definido no passo anterior); c) um conjunto de n pontos/pixels é escolhido nesse semiarco para criação de janelas/sub-imagens (Figura 5a) para busca dos novos segmentos; d) a técnica de deteção de linhas retas, usada para detetar sementes, é aplicada nessas janelas/sub-imagens; e) se existe algum segmento de reta que tenha uma extremidade coincidente/próxima da extremidade da semente, colete-o e o assuma como semente e reinicie a busca nessa nova posição dentro da imagem; f) se não existe segmento de reta com essa característica, na primeira janela, mude de janela e repita os passos "d" e "e"; g) se ocorrer a varredura das $\mathrm{n}$ janelas/sub-imagens e não for encontrado um segmento de retas com aptidão para ser rodovia, elimine as primeiras colunas (distância d na Figura 5b) da janela/sub-imagem e repita os passos "d", "e" e "f", mas agora verificando a existência de segmentos de reta não consecutivos à semente, mas que tenham características espectrais e geométricas de rodovias; h) se ainda não encontrar um segmento representativo de uma rodovia, altere o tamanho da distância d e repita "d", "e", "f" e "g"; i) se 
não existe segmento de reta encerre a busca e vá para o outro sentido do segmento semente; $j$ ) findada a busca no outro sentido, reinicie todo o processo com uma nova semente, caso exista; $\mathrm{k}$ ) no final do processo de busca, traçe o eixo detetado na imagem e encerre a atividade.

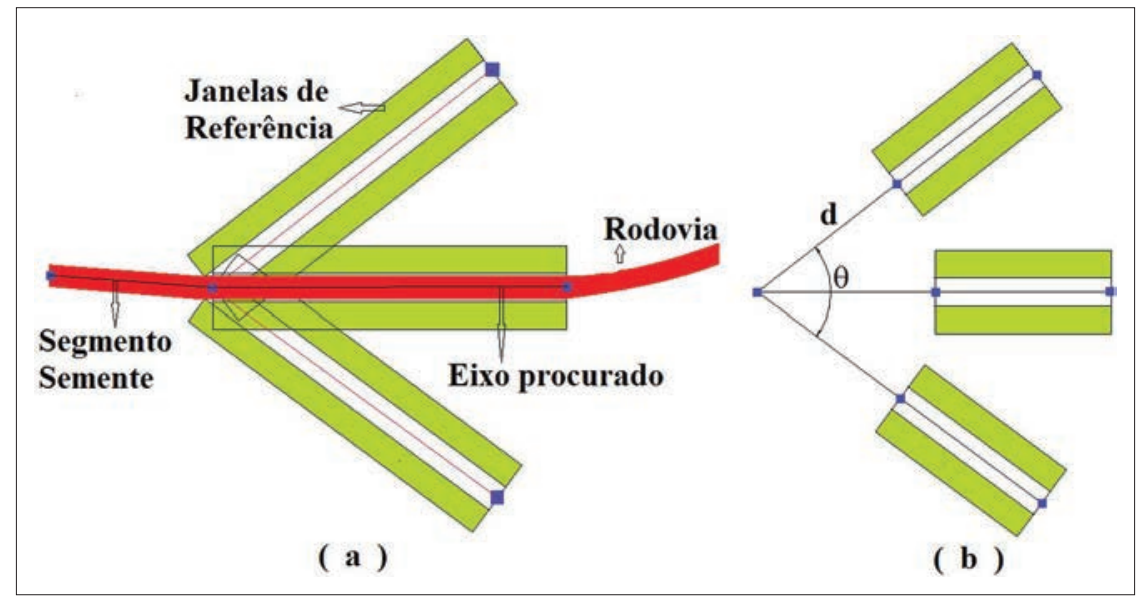

Figura 5 - Janelas/Sub-imagens de busca por novos segmentos

A análise de pertinência ou não de um segmento a uma rodovia é feita por meio de uma função de aptidão que engloba quatro parâmetros. O primeiro parâmetro dessa função analisa se um perfil transversal de uma rodovia, coletado na janela/sub-imagem (Figura 5), tem correlação espectral com um perfil transversal de referência. Esse perfil de referência tem comprimento de 3 vezes a lagura da rodovia na imagem. E a largura é definida como a média das larguras de todas as sementes detetadas. O peso é dado pelo indice de correlação entre os dois perfis.

Para evitar que o segmento procurado retorne sobre o anterior (semente), o segundo parâmetro, definido por $\theta$ (ângulo que orienta a busca de novos segmentos), força a busca por segmentos que estejam sempre à frente do atual. A medida desse parâmetro é definida como o ângulo (radianos) formado entre os dois vetores (segmento semente e novo segmento).

O terceiro parâmetro é inserido na função aptidão para analisar a resposta espectral ao longo de todo o novo segmento. Esse parâmetro é determinado por meio de uma medida de similaridade entre os valores 
digitais do segmento em questão e a média dos valores espectrais de todas as sementes. Um exemplo de função para medida de similaridade é a distância de mahalanobis.

O quarto parâmetro pondera a informação altimétrica de uma rodovia. Tem a função de ponderar o quão plana é a área ao redor do novo segmento, se tem similaridade com uma pista de rodovia real. A medida é feita pelo RMS obtido por meio de ajustamento por mínimos quadrados das altitudes existentes no plano. Esse plano é definido como a região central (faixa clara) das janelas/sub-imagens mostrada na Figura 5, com largura igual à média das larguras das sementes.

\section{RESULTADOS E DISCUSSÕES}

Nesse estudo, foi usado o cluster k-média, por ser de fácil implementação, por facilitar a incorporação de outros métodos e por apresentar resultados satisfatórios, condizentes o trabalho em questão. A matriz $\mathrm{X}$, de entrada do k-média, foi substituída pela sub-imagem, considerando suas linhas como as observações e as colunas como às variáveis. O valor de $\mathrm{k}$ foi definido empiricamente igual a 5 .

Os valores pré-definidos para os parâmetros exigidos foram: $\otimes=150$ graus (ângulo que orienta a busca de novos segmentos); Tim=11 e 21 pixels (tamanho das janelas/sub-imagens); as distâncias "d" de afastamento das janelas/sub-imagens em relação à semente foram de: Tim/2 e 2*Tim/3; T=Tim/2 (limiar que define o tamanho mínimo aceitável para seleção de segmentos semente); T1=3m (limiar que define a dispersão máxima em relação à reta de regressão para seleção de segmentos semente). Os limiares para a regressão (escolha dos segmentos semente) e para a função de aptidão (escolha dos novos segmentos) variaram entre 0,6 e 0,8 , conforme os experimentos realizados. Os valores da ponderação dos quatro parâmetros da função de aptidão foram, respetivamente:0,3, 0,2, 0,2 e 0,3. Todos os valores dos parâmetros foram definidos empiricamente, após realização de vários experimentos. O valor de T1 foi definido com base na premissa de que a largura padrão de uma rodovia é de 6 metros. Por fim, os eixos extraídos foram validados com os índices de 
completeza, correção e RMS, apresentados em Heipke et al. (1997), com os respectivos modelos (Equações 5, 6 e 7).

$$
\begin{aligned}
& \text { completeza }=\frac{L r c}{L r} \\
& \text { correção }=\frac{L d c}{L d} \\
& R M S=\sqrt{\frac{\sum_{i=1}^{n} d_{i}{ }^{2}}{n}}
\end{aligned}
$$

Onde, Lr é o comprimento total dos eixos de referência; Lrc é o comprimento dos eixos de referência detectados corretamente; Ld é o comprimento total de eixos detectados; Ldc é o comprimento de eixos detectados corretamente; e $d_{i}$ é a distância entre um pixel detectado e o pixel de referência mais próximo.

Todos os segmentos semente detetados foram plotados sobre a imagem real e analisados visualmente, em cada experimento. A maioria absoluta desses segmentos pertenciam realmente a algum tipo de rodovia. Em uma mesma rodovia foram detetados vários segmentos semente. A diversidade em uma mesma rodovia pode ser útil quando existem muitos obstáculos e/ou grandes obstruções. Nesses casos, a rodovia seria detetada, por um lado do obstáculo, usando um determinado segmento semente e, pelo outro lado, usando uma segunda semente. Em rodovias sem obstruções, teoricamente apenas um segmento semente seria suficiente para extraí-la.

\section{1. Área 1}

Para realização de uma análise quantitativa, foram realizados 12 experimentos variando os valores dos limiares usados nas funções de aptidão e o tamanho das sub-imagens, conforme Tabela 1. Por exemplo, no primeiro experimento (Experimento 1: 11-0,70-0,70-BandaRed-MDE), os valores 11, 0,70 e 0,70 referem-se ao tamanho da sub-imagem, limiar de aptidão para a deteção de segmento semente e limiar de aptidão para a deteção de novos segmentos, respetivamente. Os dados usados nesse experimento foram a banda "red" (faixa espectral) integrada com o MDE da mesma área, ambos obtidos por VANT. A escolha desses parâmetros para análise deve-se ao fato das suas interferências diretas nos resultados. O tamanho da sub-imagem afeta diretamente na deteção 
de estradas que apresentam curvas sinuosas. Quanto menor o valor do primeiro limiar, maior será o número de segmentos sementes detetados, aumentando a possibilidade de erros (deteção de falsas-sementes), onde trilhas ou pequenos caminhos podem ser percebidos como rodovias. $\mathrm{O}$ segundo limiar é o controlador da extensão da rede de rodovias extraída. Quando maior esse valor, menor o tamanho da rede extraída, e menor será a probabilidade de erros. Somado aos parâmetros, tem a qualidade espectral da imagem usado. Como a maioria das rodovias existentes nas cenas usadas não tem pavimentação, o solo exposto é predominante. Essa feição tem a propriedade de refletir mais a radiação vermelha, reduzindo para o verde, e muito menos no azul. Por esse motivo foram usadas as bandas separadamente e uma média das três (intensidade). O MDE foi utilizado como contribuinte em todos os experimentos.

Devido à quantidade de testes executados e ao tamanho das imagens, apenas dois resultados são mostrados na forma de imagem. Nessa análise foi verificada a qualidade geométrica da vetorização gerada. Os resultados apresentados na Figura 6 correspondem aos experimentos 4 (11-0,6-0,6-BandaRed-MDE) e 7 (21-0,7-0,7-BandaRed-MDE), respectivamente.

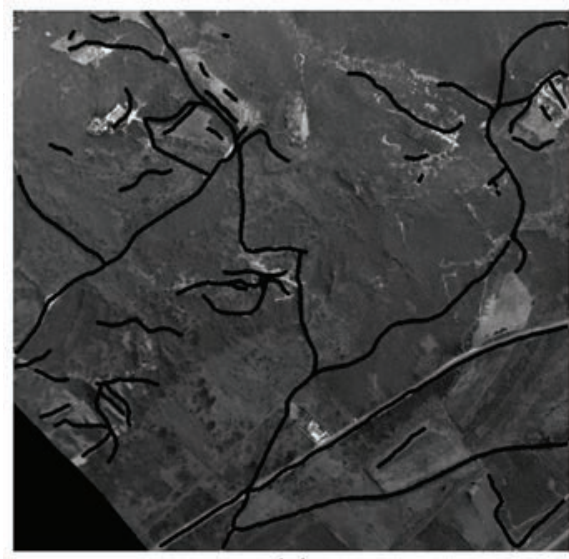

(a)

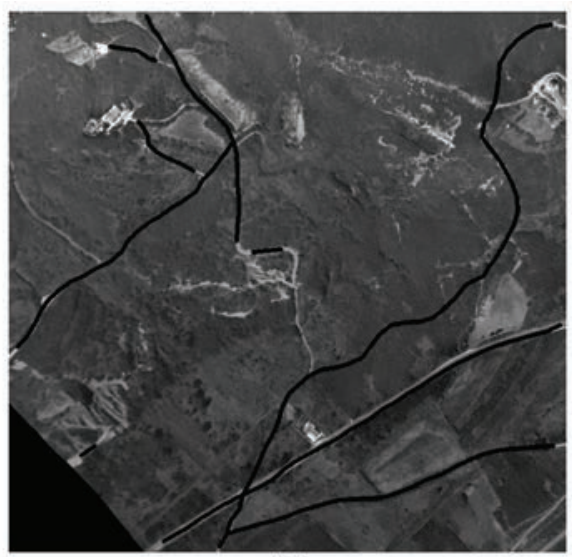

(b)

Figura 6 - Rodovias extraídas: a) experimento 7 e b) experimento 4

É notório, visualmente, que no resultado apresentado na Figura 6a todas as rodovias foram extraídas. Porém, observa-se que muitos falsos- 
-segmentos (falsa-rodovia) foram extraídos em toda a área teste, com maior concentração na região Sudoeste. Os falsos-segmentos extraídos contribuem com a acentuada redução do índice de correção (53,3\%). A rodovia asfaltada foi totalmente extraída, mas é visível os erros geométricos ocorridos. O eixo foi detectado na forma de "zig-zag" ao longo de toda a rodovia. Esse erro é responsável pela redução do índice completeza para $87,9 \%$ (Tabela 1 ), pois todas as demais rodovias foram extraídas com qualidade.

Tabela 1 - Qualidade dos eixos detectados na Área 1

\begin{tabular}{|c|c|c|c|}
\hline \multirow{2}{*}{ Experimentos } & \multicolumn{3}{|c|}{ Medidas de Acurácia } \\
\cline { 2 - 4 } & Completeza & Correção & RMS (pixel) \\
\hline 1- (11-0,70-0,70-BandaRed-MDE) & 0,656 & 0,745 & 17,078 \\
\hline 2- (11-0,75-0,70-BandaRed-MDE) & 0,720 & 0,816 & 10,951 \\
\hline 3- (11-0,80-0,70-BandaRed-MDE) & 0,578 & 0,812 & 11,715 \\
\hline 4- (21-0,70-0,70-BandaRed-MDE) & 0,691 & 0,891 & 10,847 \\
\hline 5- (21-0,75-0,70-BandaRed-MDE) & 0,577 & 0,917 & 6,824 \\
\hline 6- (21-0,80-0,70-BandaRed-MDE) & 0,450 & 0,912 & 7,663 \\
\hline 7- (11-0,60-0,60-BandaRed-MDE) & 0,879 & 0,533 & 25.081 \\
\hline 8- (11-0,60-0,70-BandaRed-MDE) & 0,798 & 0,668 & 17,759 \\
\hline 9- (11-0,60-0,80-BandaRed-MDE) & 0,566 & 0,718 & 12,936 \\
\hline 10- (11-0,75-0,70-BandaGreen-MDE) & 0,632 & 0,736 & 18,018 \\
\hline 11- (11-0,75-0,70-BandaBlue-MDE) & 0,668 & 0,775 & 19,130 \\
\hline 12- (11-0,75-0,70-Intensidade-MDE) & 0,684 & 0,780 & 15.622 \\
\hline
\end{tabular}

Ao aumentar o tamanho da sub-imagem para 21 pixels e os valores de ambos os limiares para 0,70 , manteve a qualidade geométrica das rodovias sem pavimentação, reduziu o erro de correção $(89,1 \%)$, com a eliminação dos falsos-segmentos, mas aumentou o erro de completeza $(69,1 \%)$, ao deixar de extrair trechos das rodovias nas regiões Central, Norte e Oeste da área de estudo. Embora tenha reduzido o efeito "zig-zag" na extração da rodovia pavimentada, em relação ao resultado apresentado na Figura 6a, ainda é visível o erro geométrico existente.

Nos três primeiros experimentos, alterando apenas o limiar da função de aptidão para deteção de sementes, pode-se observar que existe um ponto de máximo para os valores desse limiar (Tabela 1). Os valores foram 
configurados em 0,7, 0,75 e 0,8. Sendo mais criterioso (limiar=0,7), selecionado um número menor, porém segmentos mais refinados, o resultado foi melhor $(\approx 8 \%)$ do que aquele onde foi dada maior liberdade de seleção (limiar = 0,8). Aplicando um valor intermediário para esse limar $(0,75)$, todos os índices indicaram maior acurácia, isto é, foi extraída corretamente uma rede de rodovias maior, com menor números de falsos segmentos de rodovia e com melhor qualidade geométrica (RMS=10,951 pixels). O alto valor do RMS, de 7,55 metros, é devido aos falsos segmentos, que mesmo em pequeno número, contribuem com o erro por estarem localizados em regiões afastadas das rodovias existentes na imagem.

Mantendo fixos os dois limiares, em relação ao grupo dos três primeiros experimentos, e alterando o tamanho das sub-imagens para 21 pixels, o limiar definido para as sementes, com seu valor intermediário $(0,75)$ ainda apresentou o melhor resultado em termos de correção e RMS. Comparando com o primeiro trio de experimentos, houve um grande avanço em termos de correção e RMS nos três casos, mas o índice de completeza aponta uma redução na malha viária detetada corretamente, nos dois últimos experimentos. Isso significa que $14,3 \%$ e $12,8 \%$ das rodovias extraídas corretamente no segundo e terceiro experimentos, respetivamente, não foram extraídas no quinto e sexto experimentos. Contudo, houve uma redução dos falsos segmentos em 14,6\%, 10,1\%, 10,0\% do primeiro para o segundo trio de experimentos.

Reduzindo consideravelmente o valor do limiar da aptidão das sementes, mantendo fixo o tamanho da sub-imagem, e aumento o segundo limiar, houve ganho em completeza, mas aumentou o número de falsos segmentos detetados, de acordo com os valores de correção do terceiro trio de experimentos. Adicionalmente, o erro geométrico (RMS) também foi muito superior aos dois trios de exerimentos anteriores, superando os $100 \%$ em alguns casos. Dentro desse trio de experimentos houve um decréscimo da completeza em detrimento do aumento da correção, do sétimo para o nono experimento. Nessa configuração, o ganho em termo geométrico foi de aproximadamente $50 \%$, ao variar o valor do limiar de 0,6 para 0,8 .

Por fim, considerando o tipo de dados de entrada, alternando o MDE 
com uma das bandas referentes às faixas espectrais do vermelho, verde, azul e a com imagem intensidade, percebeu-se que com as bandas verde, azul e imagem intensidade, a dimensão da rede viária extraída corretamente foi similar, com respetivos valores de completeza: 63,2, 66,8 e $68,4 \%$. Com a banda do vermelho, a malha extraída corretamente foi 3,6\% maior. Os valores de RMS continuaram altos. Igualmente aos outros casos, isso se deve à localização longíqua dos falsos-segmentos detetados em relação as rodovias existentes.

\section{2. Área 2}

A mesma configuração utilizada nos experimentos com a Área 1 foi aplicada na imagem da Área 2. A Tabela 2 mostra os valores dos índices completeza, correção e RMS, referenciando aos erros medidos em cada experimento.

Tabela 2 - Qualidade dos eixos detetados na Área 2

\begin{tabular}{|c|c|c|c|}
\hline \multirow{2}{*}{ Experimentos } & \multicolumn{3}{|c|}{ Medidas de Acurácia } \\
\cline { 2 - 4 } & Completeza & Correção & RMS (pixel) \\
\hline 1- (11-0,70-0,70-BandaRed-MDE) & 0,707 & 0,711 & 9,799 \\
\hline 2- (11-0,75-0,70-BandaRed-MDE) & 0,710 & 0,862 & 9,705 \\
\hline 3- (11-0,80-0,70-BandaRed-MDE) & 0,553 & 0,882 & 0,550 \\
\hline 4- (21-0,70-0,70-BandaRed-MDE) & 0,605 & 0,900 & 1,438 \\
\hline 5- (21-0,75-0,70-BandaRed-MDE) & 0,276 & 0,944 & 0,610 \\
\hline 6- (21-0,80-0,70-BandaRed-MDE) & - & - & - \\
\hline 7- (11-0,60-0,60-BandaRed-MDE) & 0,921 & 0,696 & 32,713 \\
\hline 8- (11-0,60-0,70-BandaRed-MDE) & 0,871 & 0,783 & 18,615 \\
\hline 9- (11-0,60-0,80-BandaRed-MDE) & 0,479 & 0,869 & 12,820 \\
\hline 10- (11-0,75-0,70-BandaGreen-MDE) & 0,725 & 0,834 & 0,573 \\
\hline 11- (11-0,75-0,70-BandaBlue-MDE) & 0,788 & 0,871 & 0,288 \\
\hline 12- (11-0,75-0,70-Intensidade-MDE) & 0,695 & 0,861 & 0,350 \\
\hline
\end{tabular}

No primeiro grupo de experimentos (1, 2 e 3) observou-se que ao aumentar o limiar de seleção das sementes $(\mathrm{L}=0,70$ para $\mathrm{L}=0,80)$, aumentou a qualidade dos resultados em termos geométricos e de correção. Embora o ganho em correção tenha sido modesto (máximo de $2 \%$ ), o ganho geométrico foi acentuado, com RMS reduzido de aproximadamente 9,2 
pixel (6,44 metros). Por outro lado, quase $16 \%$ da rodovia de referência deixaram de ser extraídas no experimento 3, quando comparado aos resultados dos experimentos 1 e 2 .

Com o aumento do raio de busca ( $\mathrm{J}=21$ pixels) a completeza foi reduzida fortemente, o que significa que pequenos trechos das rodovias reais foram extraídos. Por outro lado, a correção aponta que mais de $90 \%$ dos eixos extraídos são verdadeiros, ou seja, pertencem a uma determinada rodovia. O máximo RMS foi de aproximadamente 1 metro. No experimento 6, o alto valor do limiar para seleção das sementes $(L=0,8)$ não permitiu a deteção de sementes nessa imagem. Isso mostra que o valor de L é dependente do tamanho do raio $\mathrm{J}$, indicando que o valor do limiar deve ser controlado ao aumentar o raio.

No terceiro grupo, ao dar mais liberdade na seleção de sementes $(\mathrm{L}=0,6)$, ocorre erros consideráveis geometricamente, excedendo os 32 pixels $(22,4$ metros). Esse fato ocorre porque aumenta a seleção de falsas sementes e, consequentemente a deteção de falsos eixos. Contudo, é percebido um ganho superior a $21 \%$ em termos de completeza, se comparada a máxima completeza desse grupo (experimento 7) com a máxima dos grupos 1 e 2 (experimento 2). A correção, nesse grupo de experimento, aumentou com o aumento do limiar de seleção de novos segmentos ( $\mathrm{L} 1=0,6$ para $\mathrm{L} 1=0,8)$, enquanto a completeza reduziu em quase metade. Assim, pode-se afirmar que ao aumentar L1 aumenta a quantidade de eixos detetados corretamente, mas aumenta o trecho de rodovia em que deixou de ser detetado.

Os melhores resultados estão no quarto grupo, nos experimentos 10, 11 e 12, onde foram mantidos fixos os limiares (L e L1) e alteradas as imagens de entrada. O resultado com a banda azul foi o melhor de todos os experimentos realizados nas duas áreas testes (Figura 7), apresentando altos valores de completeza e correção e baixo valor de RMS, o que expressa qualidade nos resultado. Com a banda verde, os resultados estão próximos daqueles obtidos com a banda azul, mas ligeiramente abaixo, com destaque para o RMS, com diferença de 19,95 centímetros. Usando a imagem intensidade (experimento 12), a falha maior foi na capacidade de detetar toda a rodovia (completeza $=69,5 \%$ ), pois a maioria do que 
foi detetado está correto (correção 86,1\%).

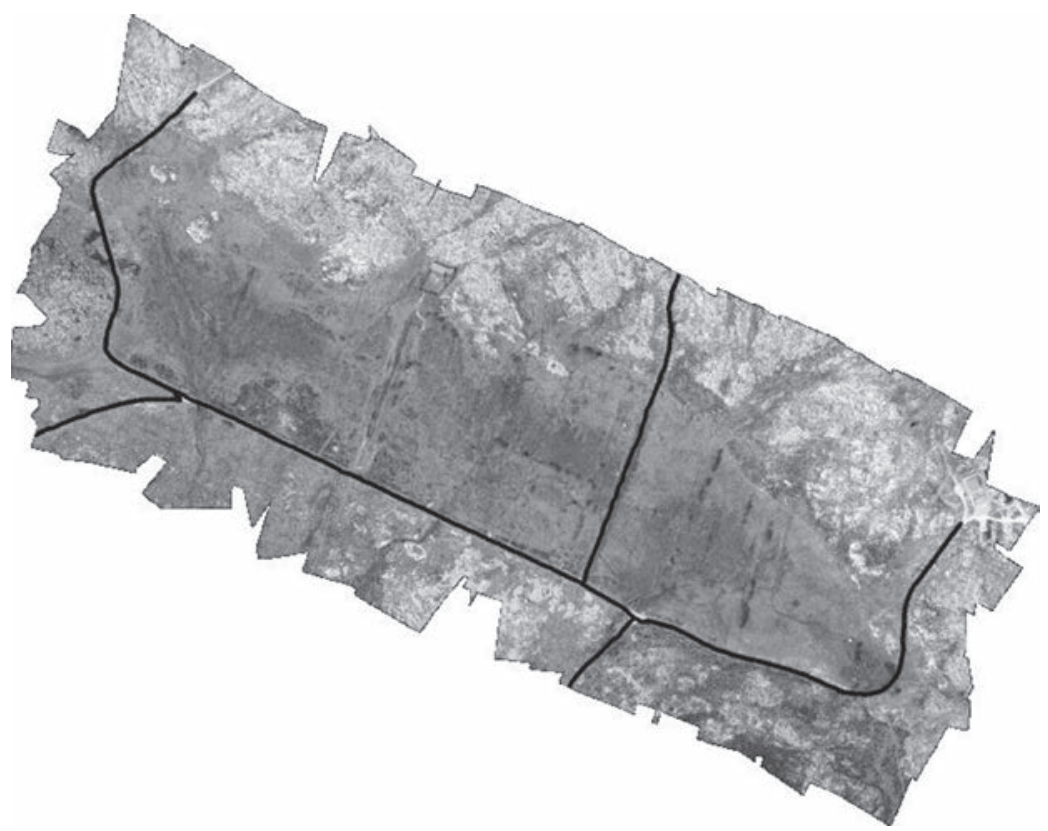

Figura 7 - Resultado usando a configuração: 11-0,75-0,7-BandaBlue-MDE

Com exceção do último grupo de experimentos (10, 11 e 12), os resultados obtidos para as duas áreas (Área 1 e Área 2) são semelhantes. Isso significa que o algoritmo teve um comportamento análogo ao manter as configurações inalteradas. Em relação ao último grupo, acredita-se que o melhor resultado (nos três experimentos) na Área 2 foi devido à qualidade dos MDEs. As imagens da Área 1 não possibilitaram a geração de um MDE com mesma qualidade que o MDE da Área 2. Uma possível explicação é a altitude de voo e o comportamento distinto das aeronaves, tais como velocidade e tempo para disparo da câmera que é bem mais rápido no caso da aeronave convencional quando comparada ao VANT.

\section{ConCluSÃo}

Nesse estudo, foi usado o k-média, que é um algoritmo de agrupamento que além de fácil implementação, tem processamento rápido (quando comparado com outros de mesma categoria) e ainda permite a incor- 
poração de outros métodos sem degradar os resultados. Os resultados obtidos nesse trabalho mostram a eficiência dessa junção de técnicas: cluster, regressão linear, deteção de segmentos.

Rodovias são feições lineares que apresentam desafios no processo de extração em imagens digitais, principalmente por apresentarem curvas sinuosas e existirem feições com características espectrais similares. $\mathrm{O}$ método proposto nesse estudo buscou resolver esse problema, englobando informações espectrais (fotos aéreas), geométricas (segmentos consecutivos e adjacentes) e altimétricas (MDE) de rodovias, no processo de extração automática. Os resultados mostram que um ganho considerável, dado que se trata de uma tarefa maçante e onerosa. Embora o tempo de processamento não seja objeto dessa pesquisa, afirma-se, com base nos experimentos realizados, que é possível vetorizar quilômetros de rodovias em poucos minutos, em um mesmo trabalho que pode demandar horas, se realizado manualmente.

Considerando a resolução das fotos, o preço de uma câmera de pequeno formato, a rapidez e o custo envolvidos no voo (e.g. aeromodelo e combustível), o espaço utilizado para decolagem e pouso e a qualidade dos resultados, pode-se afirmar que os produtos da tecnologia VANT são viáveis, em abordagens automáticas, do ponto de vista econômico, temporal e ambiental, especialmente em execuções de pequenos projetos de mapeamento rodoviário, em pequenas e médias escalas.

\section{AgRAdecimentos}

À Faculdade de Ciências do Porto pela disponibilização do espaço físico e das cenas usadas.

À CAPES (Coordenação de Aperfeiçoamento de Pessoal de Nível Superior) pelo apoio financeiro concedido.

\section{BIBLIOGRAFIA}

AGUASCA, Albert et al. (2013) - "Light-Weight CW/FM SAR Sensors for Small UAVs". Sensors, Vol. 13, 3204-3216. 
BAUMGARTNER, Albert et al. (1999) - "Automatic Road Extraction Based on MultiScale, Grouping, and Context”. Photogrammetric Engineering \& Remote Sensing (PE\&RS), Vol. 65, 777-785.

CHIANG, Kai-Wei et al. (2012) - "The Development of an UAV Borne Direct Georeferenced Photogrammetric Platform for Ground Control Point Free Applications". Sensors, Vol. 12, 9161-9180.

CRAMER, Michael (2013) - "The UAV@LGL BW Project - A NMCA Case Study". (p.165-179, Stuttgart) Disponível online no endereço url: http://www.ifp. uni-stuttgart.de/ publications/phowo13/150Cramer.pdf (acedido em 11 October, 2013).

DU, Huang \& GAO, Yang (2012) - "Inertial Aided Cycle Slip Detection and Identification for Integrated PPP GPS and INS”. Sensors, Vol. 12, 14344-14362.

FENG, Wang et al. (2009) - "UAV Borne Real-time Road Mapping System". In Proceeding of the Urban Remote Sensing Joint Event, Shanghai, China. Disponível online no endereço url: http://ieeexplore.ieee.org/xpl/articleDetails.jsp?arnumber=5137476 (acedido em 11 October, 2013).

GAN, Guojun et al. (2007) - Data clustering: theory, algorithms, and applications. SIAM books: University City Science, Philadelphia, 466 p.

GONÇALVES, Jose Alberto \& HENRIQUES, Renato (2013) - "Topographical monitoring of coastal areas with UAV imagery". In Proceeding of the 6th EARSeL Workshop on Remote Sensing of the Coastal Zone, Matera, Italy. Disponível online no endereço url: http://www.earsel.org/SIG/CZ/6th-workshop/proceedings.php (acedido em 11 October, 2013).

HEIPKE, Christian et al. (1997) - "Evaluation of Automatic Road Extraction". In Procedings IAPRS - 3D Reconstruction and Modeling of Topographic Objects, Stuttgart, germany. Disponível online no endereço url: http:// citeseerx.ist.psu.edu/viewdoc/download; jsessionid=347BDE79C49E8 7CAE69D9B97DB85906E?doi=10.1.1.30.7686\&rep=rep1\&type=pdf (acedido em 11 October, 2013).

HRUSKA, Ryan et al. (2012) - "Radiometric and Geometric Analysis of Hyperspectral Imagery Acquired from an Unmanned Aerial Vehicle. Remote Sensing, Vol. 4, 2736-2752.

HU, Xiangyun \& TAO, Vincent (2007). Automatic Extraction of Main Road Centerlines from High Resolution Satellite Imagery Using Hierarchical Grouping. Photogrammetric Engineering \& Remote Sensing, Vol. 73, № 9, 1049-1056.

LIN, Yucong \& SARIPALLI, Srikanth (2012) - "Road Detection from Aerial Imagery". In Proceedings of the International Conference on Robotics and Automation, Saint Paul/Minnesota, USA. Disponível online no endereço url: http:// www.public.asu.edu/ ylin122/road_detect.pdf (acedido em 20 April, 2013). 
LINGUA, Andrea et al. (2009) - "Performance Analysis of the SIFT Operator for Automatic Feature Extraction and Matching in Photogrammetric Applications". Sensors, Vol. 9, $\mathrm{N}^{\circ}$ 5, 3745-3766.

SILVA, Claudionor \& CENTENO, Jorge (2011) - "Semiautomatic Extraction of Main Road Centrelines in Aerial Images Acquired over Rural Areas”. International Journal of Remote Sensing, Vol. 33, No 2, 502-516.

SILVA, Claudionor et al. (2010) - "Automatic Road Extraction in Rural Areas Based on Digital Imaging and Laser Scanner Data”. Journal of Civil Engineering and Architecture, Vol. 4, $\mathrm{N}^{\circ}$ 40, 285-296.

SKOGLAR, Per et al. (2012) - "Road Target Search and Tracking with Gimballed Vision Sensor on an Unmanned Aerial Vehicle”. Remote Sensing, Vol. 4, $\mathrm{N}^{\mathrm{o}}$ 7, 2076-2111.

WALLACE, Luke et al. (2012) - "Development of a UAV-LiDAR System with Application to Forest Inventory”. Remote Sensing, Vol. 4, N 7, 1519-1543.

ZHANG, Chunsun (2008) - "An Uav-Based Photogrammetric Mapping System for Road Condition Assessment”. In: Proceedings of the IAPRS - International Archives of the Photogrammetry, Remote Sensing and Spatial Information Sciences, Beijing, China. Disponível online no endereço url: http://www. isprs.org/proceedings/XXXVII/ congress/5_pdf/109.pdf (acedido em 20 April, 2013). 
Série Documentos

Imprensa da Universidade de Coimbra

Coimbra University Press

2015

- U M

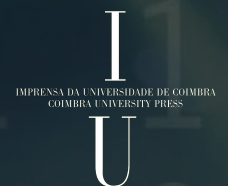

